\title{
Congenital aortic valve stenosis
}

INSERM

\section{Source}

INSERM. (1999). Orphanet: an online rare disease and orphan drug data base. Congenital aortic valve stenosis. ORPHA:3093

A rare aortic malformation of variable severity and clinical presentation. Clinical presentations range from a neonatal severe presentation often associated with sudden cardiac death, to a slowly progressive stenosis that presents later with cardiac murmur, chest pain, dizziness, and loss of consciousness with exercise-induced exacerbations. Echocardiography reveals atresia or dysplasia of the aortic valve most commonly associated with a bicuspid morphology, restricted left ventricular outflow, and left ventricular hypertrophy. 